\title{
Fe thin-film growth on Au(100): A self-surfactant effect and its limitations
}

\author{
V. Blum, Ch. Rath, S. Müller, L. Hammer, and K. Heinz \\ Lehrstuhl für Festkörperphysik, Universität Erlangen-Nürnberg, Staudtstrasse 7, D-91058 Erlangen, Germany \\ J. M. García \\ Instituto de Microelectrónica de Madrid, CNM-CSIC, E-28760 Madrid, Spain \\ J. E. Ortega \\ Departamento de Física, Universidad del País Vasco, San Sebastián, Spain
}

J. E. Prieto, O. S. Hernán, J. M. Gallego, A. L. Vázquez de Parga, and R. Miranda

Departamento de Física de la Materia Condensada C-3, Universidad Autónoma de Madrid, E-28049 Madrid, Spain

(Received 8 October 1998; revised manscript received 15 January 1999)

\begin{abstract}
The combination of low-energy electron diffraction intensity analyses and scanning tunneling microscopy was used to investigate the morphology and atomic structure of thin $\mathrm{Fe}$ films grown on $\mathrm{Au}(100)$ at $400 \mathrm{~K}$. Deposition of only about 0.2 monolayers (ML) Fe is sufficient to lift the reconstruction of the clean substrate. In the initial growth process $(\leqslant 1 \mathrm{ML})$ place exchanges between $\mathrm{Fe}$ and $\mathrm{Au}$ lead to almost two-dimensional subsurface Fe film growth with one layer of Au covering the entire film. This way, gold acts as a "selfsurfactant." Yet, there are deviations from two-dimensional growth, with a second Fe layer beginning to grow before the first one is fully completed and some substitutional disorder developing in the film because of incomplete place exchange. The amount of gold floating on the surface only gradually decreases with further increasing film thickness. At about 2 ML the surface undergoes a complete restructuring during which short "wormlike" chains of atoms form and long-range order is destroyed. Nevertheless, the existence of large terraces of little roughness proves that some surface activity of gold remains. At coverages of several ML, long-range order is reestablished with the Fe film growing in an undistorted bcc arrangement. Although parts of the film are still covered by gold, the surface morphology is not very different from that known for homoepitaxial growth of Fe on Fe(100), i.e., gold has stopped to serve as a "self-surfactant."
\end{abstract}

[S0163-1829(99)06223-2]

\section{INTRODUCTION}

Recent attention payed to ferromagnetic thin films deposited on nonmagnetic substrates (see, e.g., Ref. 1) is motivated both by the opportunity for fundamental studies of ferromagnets of restricted dimensionality and the related technological potential of corresponding multilayer structures that exhibit oscillatory magnetic coupling ${ }^{2}$ or giant magnetoresistance. ${ }^{3}$ Yet, the "tailoring" of such structures with specific morphology and atomic structure is frequently inhibited by deviations from the desired film order occurring during the film growth. The search for the "right" growth recipe can take years, ${ }^{4,5}$ in particular, when the atomic order and film morphology resulting from a certain preparation procedure is unknown. In this light, the present paper aims to contribute to the field by providing this knowledge for the epitaxial system $\mathrm{Fe} / \mathrm{Au}(100)$, which has already been subject to a number of studies focusing on both structural features (for a recent overview see Ref. 6) and magnetic properties.

The special interest in $\mathrm{Fe} / \mathrm{Au}(100)$ stems from the small lattice mismatch of about $0.6 \%$ between (bcc) $\mathrm{Fe}(100)$ and (fcc) $\mathrm{Au}(100)$. This should allow one to study the transition from the two-dimensional (2D) limit of a single (100) monolayer (ML) to quasi-3D bcc-Fe films and the growth of $[\mathrm{Au} / \mathrm{Fe}]_{n} / \mathrm{Au}(100)$ multilayers exhibiting, e.g., oscillatory magnetoresistance $^{7-9}$ and novel magneto-optic proper- ties. ${ }^{10,11}$ Even films with chemically alternating layers could be prepared $^{12,13}$ corresponding to an artificial tetragonal AuFe compound of $L 1_{0}$ structure not existing in equilibrium since $\mathrm{Au}$ and $\mathrm{Fe}$ fail to mix at room temperature. ${ }^{14}$

In earlier work ${ }^{15}$ it was retrieved that upon Fe deposition $\mathrm{Au}$ atoms segregate to the very film surface up to considerable $\mathrm{Fe}$ coverages, a finding repeatedly confirmed in subsequent work. ${ }^{6,16-21}$ Because of the much higher surface free energy of $\mathrm{Fe}\left[2.9\left(\mathrm{~J} / \mathrm{m}^{2}\right)\right]$ compared to $\mathrm{Au}\left[1.6\left(\mathrm{~J} / \mathrm{m}^{2}\right)\right],{ }^{22}$ one would expect 3D growth for $\mathrm{Fe}$ on $\mathrm{Au}(100)$. To explain the low-dimensional or even layer-by-layer growth observed instead, ${ }^{6,15,20,23-26}$ a "self-surfactant" effect of $\mathrm{Au}$ was proposed $^{16,17,20}$ in a phenomenological way, in the sense that substrate atoms floating on the surface (and not atoms of a third chemical species) account for the observed growth mode of $\mathrm{Fe}$ films on $\mathrm{Au}(100)$. Of course, for a microscopic understanding of this phenomenon a detailed knowledge of both the morphology and the atomic arrangement of the films is crucial. This is the objective of the present paper.

The film properties are known to depend significantly on preparational details, in particular, the growth temperature. In order to study specifically the proposed "self-surfactant" effect, we focus on $\mathrm{Fe} / \mathrm{Au}(100)$ films grown under one welldefined set of conditions. For room temperature deposition, authors agree that above a certain $\mathrm{Fe}$ coverage $\mathrm{Au}$ is no longer present on top of the film..$^{6,16-19,23}$ However, the value 
of this critical coverage varies in the literature, extending up to $45 \mathrm{ML}$ equivalents. ${ }^{17}$ At elevated temperatures (370 K to $470 \mathrm{~K}$ ), floating $\mathrm{Au}$ was reported even after evaporation of more than $50 \mathrm{ML} \mathrm{Fe} .^{15,20,23}$ For even higher temperatures [above $420 \mathrm{~K}$ (Ref. 27)] Au and Fe start to interdiffuse, with Fe eventually dissolving in the bulk above $520-570 \mathrm{~K} .{ }^{15,18}$ Guided by these experiences, we chose a deposition temperature of $400 \mathrm{~K}$ for the present study. This allows for the fast segregation of Au but should still inhibit substantial dissolution of $\mathrm{Fe}$ in the Au bulk. To investigate the films, we used scanning tunneling microscopy (STM) for morphological studies and quantitative low-energy electron diffraction (LEED) for the retrieval of the atomic structure. The combination of the two methods has proven to be a highly efficient alliance in earlier work. ${ }^{28}$

In the following, we demonstrate that after an initial stage of relatively flat epitaxial film growth (up to about $1 \mathrm{ML} \mathrm{Fe}$ coverage) a dramatic decrease of long-range order takes place in the range around 2-5 ML caused by a restructuring of the surface into an array of short chains ("worms"). With further increasing thickness, bcc-Fe films develop. They are still to a large extent covered by a single layer of Au but exhibit a morphology not very different from that reported for the homoepitaxial growth of $\mathrm{Fe}$ on $\mathrm{Fe}(100) .{ }^{29,30}$

\section{EXPERIMENTS AND LEED INTENSITY CALCULATIONS}

The experiments were carried out in two separate UHV vessels, both provided with a rearview LEED optics. One chamber was additionally equipped with a homemade scanning tunneling microscope as described elsewhere. ${ }^{31}$ Quantitative LEED $I(E)$ spectra were preferentially taken in the other apparatus as this allowed for more precise sample alignment and low temperatures $(100 \mathrm{~K})$ giving access to a larger and higher quality database. Both chambers contained facilities for Auger electron spectroscopy (AES), ion sputtering, and metal evaporation. The $\mathrm{Au}(100)$ crystal was prepared by mechanically polishing followed by short cycles of sputtering $\left(\mathrm{Ar}^{+}, 500 \mathrm{eV}, 20 \mu \mathrm{A} / \mathrm{cm}^{2}\right)$ and annealing until the $(5 \times 20)$ reconstruction appeared both in LEED and STM and no contaminants remained in AES. Iron was deposited from an electron bombarded high-purity Fe plate at a rate of about $0.1 \mathrm{ML} / \mathrm{min}$ (STM chamber) or from a directly heated high-purity Fe wire with rates of $0.5-1 \mathrm{ML} / \mathrm{min}$ (LEED chamber). During deposition the substrate temperature was kept at $400 \mathrm{~K}$ as controlled by W-W Re thermocouples. The use of AES allowed for the easy comparison of the Fe coverage in different experiments. The signals measured in each case are consistent with the elemental distribution within the surface as derived in the quantitative LEED analyses. For the latter, $I(E)$ spectra were measured at $100 \mathrm{~K}$ using a videobased and commercially available automated image dataacquisition system described in detail elsewhere. ${ }^{32,33}$ Normal incidence of the primary beam was adjusted by quantitative comparison of symmetrically equivalent spectra via the Pendry $R$ factor. ${ }^{34}$ Eventually the equivalent spectra were averaged in order to improve the data quality with respect to noise, inhomogeneities of the luminescent screen, or some possible residual sample misalignment. In order to allow for comparison of films prepared in the different chambers, $I(E)$ spectra were also taken in the STM chamber.

For the full dynamical intensity calculations standard computer codes were used. ${ }^{35}$ The upper energy limit of $E$ $=500 \mathrm{eV}$ and scatterers as strong as Au required the use of up to 13 relativistically calculated and spin-averaged atomic phase shifts. Electron attenuation was considered by a constant imaginary part of the inner potential fixed at $V_{o i}$ $=5 \mathrm{eV}$. Substitutional disorder was modeled using the average $t$-matrix approximation (ATA). ${ }^{36}$ The coexistence of different structural domains on the surface was treated by weighted summation of their intensities, neglecting possible coherence between electron waves diffracted from different areas. For the retrieval of the respective domain areas and the corresponding structural parameters, we used a full dynamical version of a search algorithm developed earlier in our group for tensor LEED (Ref. 37) whereby the Pendry $R$ factor $^{34} R$ serves as a measure to compare experimental and calculated $I(E)$ data. Its variance $R R=R_{\min } \sqrt{8 V_{o i} / \Delta E}$ with $\Delta E$ the energy width of the database was applied to estimate the statistical errors as usual.

\section{FILM MORPHOLOGY AS A FUNCTION OF COVERAGE}

As well known, the clean $\mathrm{Au}(100)$ surface is reconstructed exhibiting an (almost) incommensurate quasihexagonal overlayer residing on bulklike layers below ${ }^{38-40}$ leading to a superstructure described as $(5 \times 20)$, ( 5 $\times 26), c(26 \times 68)$, or simply, "hex." As reported in detail elsewhere, ${ }^{41} \mathrm{Fe}$ atoms deposited at room temperature diffuse over the reconstructed surface until they reach specific locations in the unit cell where the tensile stress is at maximum and where surface incorporation of Fe takes place. The Au atoms exchanged form new islands, which, initially, also exhibit the "hex" reconstruction. With increasing coverage, unreconstructed areas appear within the islands, attributed to $\mathrm{Au}$ atoms residing on Fe. These unreconstructed areas spread over the entire surface with further increasing coverage until (at room temperature) the reconstruction is almost completely lifted after deposition of only about $0.2 \mathrm{ML}$ of Fe. ${ }^{41}$ Consistently and in agreement with the literature, $6,15,17,18,23$ we observe that the LEED pattern switches to $(1 \times 1)$ periodicity around this coverage. Related investigations by scanning tunneling spectroscopy have shown that almost all the atoms detected at the very surface are Au atoms. ${ }^{41}$

\section{A. Low coverage: Flat growth}

Figure 1 shows snapshots of the surface morphology after deposition of increasing amounts of $\mathrm{Fe}$ at $400 \mathrm{~K}$. For low coverages $\Theta$ [Fig. 1(a), $\Theta \approx 0.15$ ML's] the surface is characterized by small unreconstructed islands and large, monolayer-high areas exhibiting a corrugation typical for the substrate reconstruction. This resembles the situation found recently for room-temperature deposition: ${ }^{42}$ Both large and small islands consist of $\mathrm{Au}$ atoms ejected to the surface in an exchange process with the $\mathrm{Fe}$ atoms deposited. While the reconstructed islands correspond to $\mathrm{Au}$ atoms residing on the $\mathrm{Au}$ substrate, the unreconstructed areas reveal the presence of $\mathrm{Fe}$ underneath. With the coverage increasing to about $\Theta$ $=0.5 \mathrm{ML}$ [Fig. 1(b)], the islands start to coalesce. Traces of 

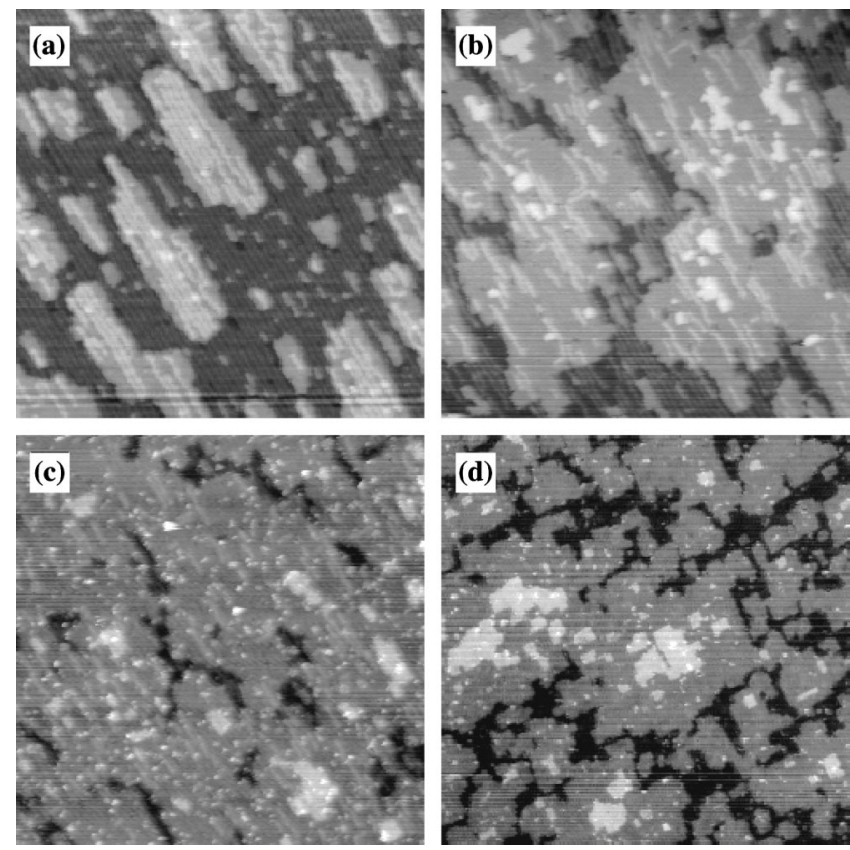

FIG. 1. STM images of the surface morphology $(850 \AA$ $\times 850 \AA$ ) during the early stages of growth: (a) $\approx 0.15$, (b) 0.5 , (c) just below 1, and (d) 1.5 ML of Fe on $\mathrm{Au}(100)$.

the distorted "hex" reconstruction can still be seen in the substrate. The unreconstructed areas have spread to cover most of the islands, although some ridgelike structures, reminiscent of the surface reconstruction, are still present on them.

Slightly below the completion of the first monolayer [Fig. $1(\mathrm{c})]$ the second level begins to be populated. Although rather flat on a large scale, the surface shows a large density of irregularities on the atomic scale and some leftovers of the ridges of the "hex" reconstruction indicating the presence of some Au both in the first and the second level. A still visible small fraction of the substrate is unreconstructed. The islands in Figs. 1(a) and 1(b) have an apparent height of about $2.0 \AA$ when measured from top to top of the respective corrugations, very close to the interplanar distance in bulk $\mathrm{Au}$. Second level islands have a height of about $1.8 \AA$, similar to islands developing in the third level after deposition of about 1.5 ML of Fe [Fig. 1(d)]. The latter exhibit no traces of the "hex" reconstruction, and the surface as a whole shows bidirectional features instead of the uniaxial arrangement in Figs. 1(a)-1(c). The LEED patterns from the surfaces displayed in Figs. 1(b) $-1(d)$ are of $(1 \times 1)$ symmetry with sharp spots, though some diffuse background intensity is always present. Furthermore, the $I(E)$ spectra characteristic for the states given in Figs. 1(c) and 1(d) are very similar.

\section{B. Intermediate stage: Dispersing the long-range order}

Deposition of about 3 ML Fe causes a sudden change in the short-range structure of the film though on a large scale [1700 $₫ 1700 \AA$, Fig. 2(a)] the surface morphology does not look very different from that presented in the previous section, with three or four atomically flat domains of different height. However, a zoomed image [Fig. 2(b)] shows that the surface consists of a large number of short chains ("worms") with an apparent height of about $\Delta h \approx 0.6 \AA$
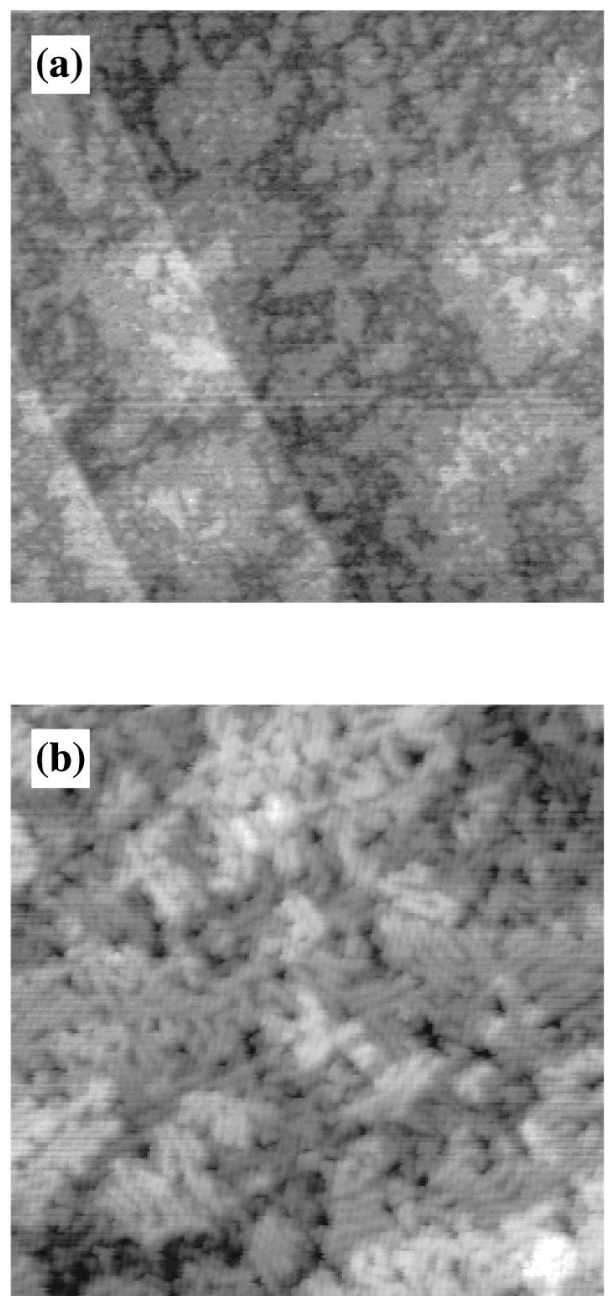

FIG. 2. (a) STM image of a 3 -ML Fe film, $1700 \AA \times 1700 \AA$ size. At this scale, the surface looks rather flat, similar to the lower coverage films. (b) Zoomed image, $600 \AA \times 600 \AA$. The surface no longer appears atomically flat, instead is covered by short elongated structures.

above the neighboring areas. Ion scattering experiments indicate that $\mathrm{Au}$ is still segregating efficiently to the external surface. ${ }^{20}$ In most cases the chains are roughly collinear but clearly exhibit no long-range order. Consistently, discrete LEED spots have disappeared as also observed earlier. ${ }^{17,27}$ With the surface in STM still appearing flat on a larger scale, the absence of the LEED pattern is surprising because of contributions to be expected from the ordered Au bulk below the "worms." Therefore, we must conclude that the "wormlike" restructuring destroys the long-range order of several layers in the film as well as in the substrate.

\section{High coverage}

Evaporation of more than $5 \mathrm{ML} F e$ makes the $(1 \times 1)$-LEED pattern reappear. Yet, in contrast to the "thinfilm" regime, the spots characteristic for this "thick film" are now relatively broad, indicating a reduced size of ordered domains. Figure 3(a) shows the STM image of a film after a deposition time of $60 \mathrm{~min}$, compared to $5 \mathrm{~min}$ for the "thin film" of Fig. 1(d). The growth is still rather flat, i.e., the difference between the lowest and highest areas found in 

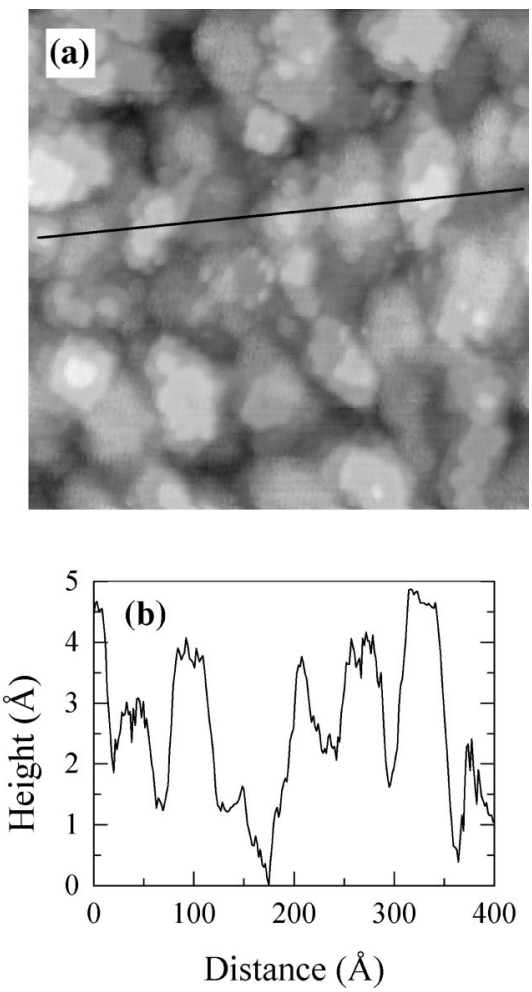

FIG. 3. (a) STM image of a thick Fe film after 60 min evaporation time, $415 \AA \times 415 \AA$. (b) Line scan along the profile indicated in (a).

STM line scans as displayed in Fig. 3(b) does not exceed $5 \AA$ corresponding to 3-4 Fe(100) interlayer distances (1.43 $\AA$ ). However, with $\sim 30 \AA$ the average terrace width has decreased significantly with respect to the case of the thin or even the "wormlike" film, consistent with earlier LEED spot profile analyses. ${ }^{25,27}$ The terraces remain atomically flat, though a quantitative evaluation of "step heights" is no longer straightforward. The overall surface morphology is similar to that observed for $\mathrm{Fe} / \mathrm{Fe}(100)$ homoepitaxy. ${ }^{29,30}$

\section{THE ATOMIC ARRANGEMENT IN THE FILM}

With the morphological information from the previous section at hand, we performed LEED $I(E)$ analyses for the two thickness ranges exhibiting $(1 \times 1)$ patterns, i.e., for the ML regime and the "thick-film" limit. Two data sets were available for each regime, one taken at $100 \mathrm{~K}$ in the LEED chamber and one at $300 \mathrm{~K}$ in the STM chamber. Although the $300 \mathrm{~K}$ sets are of lower quality due to experimental restrictions, they were analyzed to ensure the films imaged in STM correspond to the same structural states as those the LEED intensity analyses are based upon. In the following, we focus on the analysis of the higher quality $100 \mathrm{~K}$ data, not addressing the results for the $300 \mathrm{~K}$ data any further since they were practically the same within the limits of error in each case.

\section{A. Low coverage: Buried Fe}

The $100 \mathrm{~K}$ data set for the $1-\mathrm{ML}$ coverage regime was taken from a film similar to the one visualized in Fig. 1(c). It includes spectra for five symmetrically inequivalent beams between $100 \mathrm{eV}$ and $500 \mathrm{eV}$, yielding $\Delta E=1240 \mathrm{eV}$. For the structural search, already the STM images suggest considerable modifications with respect to the structural models published. So far, studies of the film geometry in the monolayer range were either restricted to the topmost $\mathrm{Au}-\mathrm{Fe}$ interlayer spacing $^{6,18}$ or assumed the surface to consist of only a single domain corresponding to a long-range ordered stacking sequence. ${ }^{17}$ By the STM images of Sec. III A, the latter assumption seems unrealistic as at least unreconstructed $\mathrm{Au}(100)$ and second layer $\mathrm{Au}$-covered $\mathrm{Fe}$ islands coexist. Nevertheless, in a first step, we performed a fit assuming only a single domain as a link to the treatment in the literature. In this step, the chemical identity of atoms in the topmost four layers $(\mathrm{Fe}$ or $\mathrm{Au}$ ) was varied, giving a total of 16 different stacking sequences, thus treating the Fe coverage as an independent fitting parameter. Additionally, the top three layer spacings were allowed to deviate up to $\pm 0.3 \AA$ from the corresponding "hard sphere" (hs) values derived from elemental bulk nearest-neighbor distances $\left(d_{\mathrm{Fe}-\mathrm{Fe}}^{\mathrm{hs}}=1.43 \AA\right.$, $d_{\mathrm{Au}-\mathrm{Fe}}^{\mathrm{hs}}=1.74 \AA, d_{\mathrm{Au}-\mathrm{Au}}^{\mathrm{hs}}=2.04 \AA$ ). Thermal vibrational amplitudes for bulk and surface atoms were adjusted independently both for $\mathrm{Fe}$ and $\mathrm{Au}$. The lateral lattice parameter was kept fixed at the value for $\mathrm{Au}(100), a=2.88 \AA$. In agreement with Begley et al., ${ }^{17}$ the best fit of all 16 chemical layer combinations tried was achieved for a single Fe layer sandwiched by the unreconstructed $\mathrm{Au}(100)$ substrate and a single Au top layer. Yet, with $R=0.39$ the quality of the fit leaves much room for improvement, i.e., an ordered singledomain model obviously does not fully meet reality, consistent with the STM images. In a second step, we additionally allowed for the presence of patches of uncovered and unreconstructed $\mathrm{Au}(100)$. The new best fit results for about $30 \%$ of the ordered surface uncovered but with $R=0.30$ is still not satisfying when compared to LEED analyses of other epitaxial systems. ${ }^{43-45}$ Therefore, we tested two kinds of deviations from this growth mode, namely that small areas of a second Fe layer form before the first one is complete and that substitutional disorder might develop during film growth. While the STM images already indicate the first deviation, the latter one is highly probable because of the place exchanges between $\mathrm{Fe}$ and Au necessary to form a buried $\mathrm{Fe}$ layer. Moreover, $\mathrm{Fe}-\mathrm{Au}$ exchanges in deeper layers cannot a priori be excluded either since interdiffusion is known to become significant at temperatures above $420 \mathrm{~K}^{27}$

It turns out that both modifications yield a clear improvement of the fit while only negligibly affecting the structure derived for the domains already considered in the previous steps. The presence of a second Fe layer, as tested by allowing for an additional domain with $\mathrm{Au} / \mathrm{Fe} / \mathrm{Fe} / \mathrm{Au}(100)$ stacking, improves the fit to $R=0.24$. This is significant in view of a variance of $R R=0.04$. With the additional evidence from the STM images, we can conclude that indeed a second layer of $\mathrm{Fe}$ begins to form before the first one is completed and before the fundamental restructuring of the surface to the "wormlike" phase takes place. Further significant improvement results when allowing substitutional disorder in the top three layers of the two Fe-covered domains, resulting in $R$ $=0.19(R R=0.03)$, which is our final best fit achieved. Its stepwise improvement is visualized in Fig. 4, which compares experimental and calculated data for two selected beams with the beam-specific $R$ factors given. In total, as 

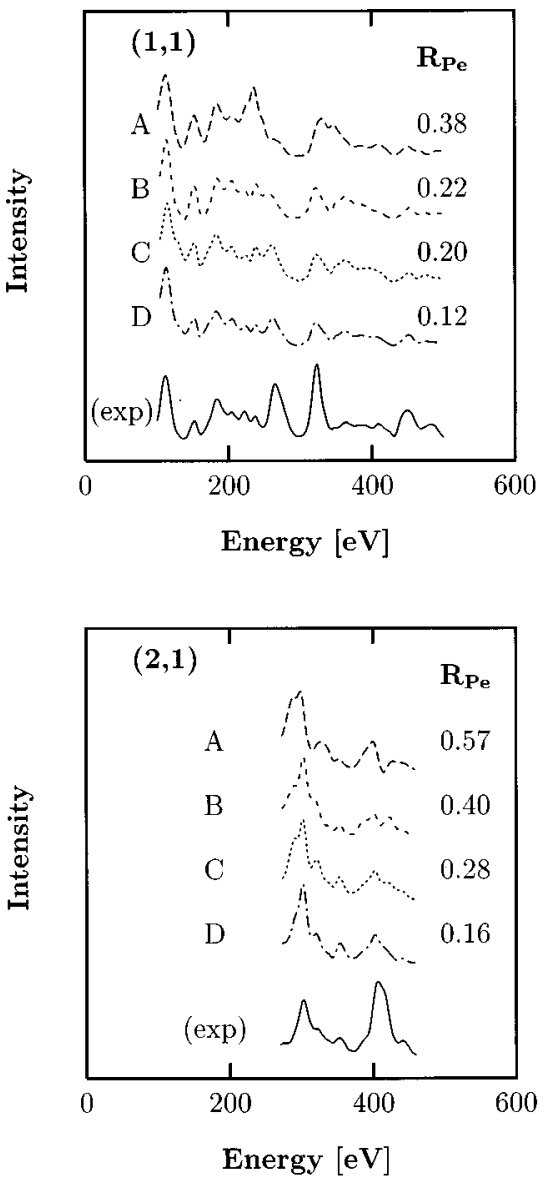

$\mathrm{A}: \mathrm{Au} / \mathrm{Fe} / \mathrm{Au}(100)$
$\mathrm{B}: \mathrm{Au}(100)+\mathrm{Au} / \mathrm{Fe} / \mathrm{Au}(100)$
$\mathrm{C}: \mathrm{Au}(100)+\mathrm{Au} / \mathrm{Fe} / \mathrm{Au}(100)+\mathrm{Au} / \mathrm{Fe} / \mathrm{Fe} / \mathrm{Au}(100)$
$\mathrm{D}: \mathrm{Au}(100)+\mathrm{Au} / \mathrm{Fe} / \mathrm{Au}(100)+\mathrm{Au} / \mathrm{Fe} / \mathrm{Fe} / \mathrm{Au}(100)+$ film disorder

FIG. 4. Best-fit LEED $I(E)$ spectra of the $(1,1)$ and $(2,1)$ beams compared to the experimental curves.

many as 17 structural and chemical parameters have been determined. This has to be compared with the available database. With $\Delta E=1240 \mathrm{eV}$ and an average peak separation of $4 V_{o i}=20 \mathrm{eV}$, it amounts to about 60 largely independent data points equivalent to a data redundancy of more than 3 , so that our fit procedure seems to be reliable.

TABLE I. Best-fit parameters for the $\sim 1$ ML film. The model includes areas covered with 0 , one or two layers of $\mathrm{Fe}$, and allows for substitutional disorder in the Fe-covered parts.

\begin{tabular}{lccc}
\hline \hline & $\mathrm{Au}(100)$ & $\mathrm{Au} / \mathrm{Fe} / \mathrm{Au}(100)$ & $\mathrm{Au} / \mathrm{Fe} / \mathrm{Fe} / \mathrm{Au}(100)$ \\
\hline Surface fraction & $15 \pm 10 \%$ & $65 \pm 15 \%$ & $20 \pm 15 \%$ \\
$d_{12}$ & $1.96 \pm 0.05 \AA$ & $1.92 \pm 0.04 \AA$ & $1.86 \pm 0.20 \AA$ \\
$d_{23}$ & $2.02 \pm 0.05 \AA$ & $1.84 \pm 0.03 \AA$ & $1.49 \pm 0.08 \AA$ \\
$d_{34}$ & $2.00 \pm 0.07 \AA$ & $2.06 \pm 0.03 \AA$ & $1.98 \pm 0.08 \AA$ \\
$c_{1}(\mathrm{Au})$ & & $100_{-}^{(+)} 15 \%$ & $100_{-}^{(+)} 40 \%$ \\
$c_{2}(\mathrm{Fe})$ & & $80_{-30}^{+20} \%$ & $70_{-70}^{+30} \%$ \\
$c_{3}(\mathrm{Fe})$ & & $20 \pm 20 \%$ & $70_{-60}^{+30} \%$ \\
$a_{v i b}($ top $\mathrm{Au})$ & $0.11 \AA$ & $0.19 \AA$ & $0.19 \AA$ \\
$a_{v i b}(\mathrm{bulk} \mathrm{Au})$ & $0.11 \AA$ & $0.11 \AA$ & $0.11 \AA$ \\
$a_{v i b}(\mathrm{Fe})$ & & $0.15 \AA$ & $0.15 \AA$ \\
\hline \hline
\end{tabular}

$\begin{array}{ccc}15 \% & 65 \% & 20 \% \\ \mathrm{Au}(100) & \mathrm{Au} / \mathrm{Fe} / \mathrm{Au}(100) & \mathrm{Au} / \mathrm{Fe} / \mathrm{Fe} / \mathrm{Au}(100)\end{array}$

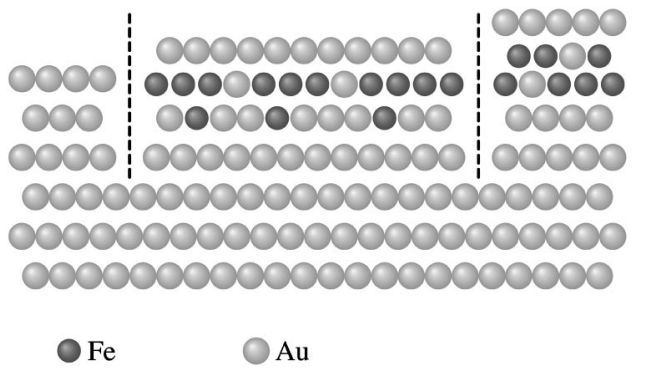

FIG. 5. Structural model of the $\mathrm{Fe} / \mathrm{Au}(100)$ thin film as given in Table I and discussed in the text. $15 \%$ of the film are not yet covered by $\mathrm{Fe}$, while on $20 \%$ of the surface a second layer of $\mathrm{Fe}$ is already present. Growth proceeds with some residual substitutional disorder in the film and some Fe situated below the actual film.

Table I summarizes the overall best-fit parameters retrieved; the corresponding structure is illustrated in Fig. 5. The Fe film covers about $85 \%$ of the Au substrate, while $15 \%$ of the surface consist of clean $\mathrm{Au}(100)-(1 \times 1)$ areas. In $20 \%$ of the surface a second Fe layer has begun to form on top of the first one. Within our limits of error $( \pm 3 \%)$, the layer spacings retrieved for the uncovered $\mathrm{Au}(100)$ patches $\left(\Delta d_{12} / d_{0}=-4 \%, \Delta d_{23} / d_{0}=-1 \%\right.$ with $d_{0}=d_{\mathrm{Au}-\mathrm{Au}}^{\mathrm{hs}}=2.04$ $\AA$ ) agree with other work in which they have been determined experimentally for a gold surface deliberately contaminated to remove reconstruction $\left[\Delta d_{12} / d_{0}\right.$ $=-2 \%, \Delta d_{23} / d_{0}=-2.8 \% \quad($ Ref. 46) $]$ or theoretically $\left[\Delta d_{12} / d_{0}=-1 \%\right.$ (Ref. 47)]. Also, there is close agreement with results for the very similar metastable $\operatorname{Ir}(100)-(1 \times 1)$ surface for which $\Delta d_{12} / d_{0}=-4 \%$ has been determined. ${ }^{48}$ For the $\mathrm{Au} / \mathrm{Fe} / \mathrm{Au}(100)$ domain there is also agreement with earlier work, though this, as mentioned, was based on the assumption of single domain models. ${ }^{17,18}$ This is reasonable as this domain clearly dominates over the others; a fact responsible for the finding that in our analysis the structural parameters only negligibly changed when new domains were included. The $\mathrm{Au}-\mathrm{Fe}$ spacings derived in the present analysis, $d_{12}=1.92 \AA$ and $d_{23}=1.84 \AA$, which are noticeably expanded compared to $d_{\mathrm{Au}-\mathrm{Fe}}^{\mathrm{hs}}=1.74 \AA$, are consistent with $d_{12}$ $=1.85 \AA$ and $d_{23}=1.825 \AA$ by Begley et al. ${ }^{17}$ and with $d_{12}$ $=1.82 \AA$ by $\mathrm{He}$ and Wang. ${ }^{18}$ Yet, there is strong disagreement to the value of $d_{12}=1.71 \AA$ retrieved by Opitz et al. ${ }^{6}$ using $\mathrm{x}$-ray photoelectron diffraction (XPD) in the forwardscattering approximation. Note that the value reported for the spacings within the artificial tetragonal FeAu compound already mentioned is $1.92 \AA$ (Ref. 13) and that the Fe-Au spacings retrieved in the present analysis are rather close to that. Within the limits of error, the spacing between the two Fe layers in the $\mathrm{Au} / \mathrm{Fe} / \mathrm{Fe} / \mathrm{Au}(100)$ domain is quite near to the value for bulk iron $\left(d_{\mathrm{Fe}-\mathrm{Fe}}^{\mathrm{hs}}=1.43 \AA\right.$ ). Its slight expansion indicated by the numerical values is consistent with the results for similarly sandwiched Fe films obtained by XPD (Ref. 6) and in Fe/Au multilayers by x-ray diffraction (XRD). ${ }^{49}$ The reproduction of such a strongly reduced value compared to $d_{\mathrm{Fe}-\mathrm{Au}}^{\mathrm{hs}}$ provides additional support for the presence of domains with a second Fe layer. Regarding the chemical parameters, we find that the topmost layer exclusively consists 
of $\mathrm{Au}$ in both $\mathrm{Fe}$ containing domains, in accordance with the strong segregation tendency of $\mathrm{Au}$ found by most previous works. In contrast, substitutional disorder with an overall substitution of $20-30 \%$ is indicated by the fit in both the second and third layer, [i.e., in the case of the $\mathrm{Au} / \mathrm{Fe} /$ $\mathrm{Au}(100)$ domain], it is not confined to the sandwiched $\mathrm{Fe}$ layer, but also extends to the Au layer immediately below. Of course, the short range order properties of the film must be kept in mind when interpreting these results. Due to the different atomic radii of Fe and $\mathrm{Au}(1.44 \AA$ and $1.26 \AA$, respectively), sizable lattice distortions in the presence of substitutional disorder must be expected. However, we are presently not able to appropriately model this property, leaving the analysis with only the retrieval of average geometrical and chemical quantities as given above. We also include the optimized rms thermal vibrational amplitudes in Table I. A value of $a_{v i b}($ bulk $\mathrm{Au})=0.11 \AA$ fits well to a bulk Debye temperature $\Theta_{D}(\mathrm{Au})=165 \mathrm{~K}$, and higher values for surface and film vibrations are reasonable. Yet, these values are rather uncertain due to the high total number of parameters in the model.

The presence of substitutional disorder sheds new light on the expanded Au-Fe spacing reported here and by other authors. ${ }^{17,18}$ It is well possible that this expansion is not inherent to the $\mathrm{Au}-\mathrm{Fe}$ stacking sequence but rather enhanced or even caused by $\mathrm{Au}$ atoms incorporated in the $\mathrm{Fe}$ layer. If that were the case, quantitative differences between interlayer spacings reported in the literature for $\mathrm{Fe} / \mathrm{Au}(100)$ in the $\mathrm{ML}$ range might easily be understood in terms of different degrees of substitutional disorder. A closer view at the $L 1_{0}$ compound produced by alternating evaporation of $\mathrm{Au}$ and $\mathrm{Fe}$ monolayers onto $\mathrm{Au}(100)$ (Ref. 13) adds an interesting facet to this interpretation. The lattice parameter of this compound as determined by XRD is $c=3.83 \AA$ perpendicular to the layers, i.e., the interlayer distance between $\mathrm{Au}$ and $\mathrm{Fe}$ layers amounts to $d_{\mathrm{Au}-\mathrm{Fe}}=1.92 \AA$. This value is not only larger than $d_{\mathrm{Au}-\mathrm{Fe}}^{\mathrm{hs}}=1.74 \AA$ but also clearly different from that predicted by theoretical calculations for such a AuFe $L 1_{0}$ compound, $d_{\mathrm{Au}-\mathrm{Fe}}=1.77 \AA$ (Ref. 50) and $1.79 \AA{ }^{51}$ respectively. However, it comes close to $d_{12}=1.92 \pm 0.04 \AA$ and $d_{23}=1.84$ $\pm 0.03 \AA$ determined for the partially disordered, sandwiched Fe layer above (Table I). With a long range order parameter of $S=0.3$, the XRD analysis of the AuFe compound ${ }^{13}$ certainly also suggests considerable disorder. Thus, as in our case, substitutional disorder rather than the features of the fully ordered structure may be held responsible for the large experimental interlayer spacing of the AuFe compound.

In conclusion, we have shown that a simple film model consisting of only one or two long-range ordered domains in the surface cannot reproduce all details of the surface structure. We have strong evidence that this is due to deviations from perfect monolayer growth of the Fe film developing below the segregated Au layer. These deviations are at least twofold: There is beginning growth of a second Fe layer before the completion of the first, and there is substitutional disorder present in the film.

\section{B. High coverage: Floating gold}

In the "thick-film" limit, i.e., after evaporation of more than $10 \mathrm{ML}$ of $\mathrm{Fe}$, the $100 \mathrm{~K}$ data set to be analyzed con-
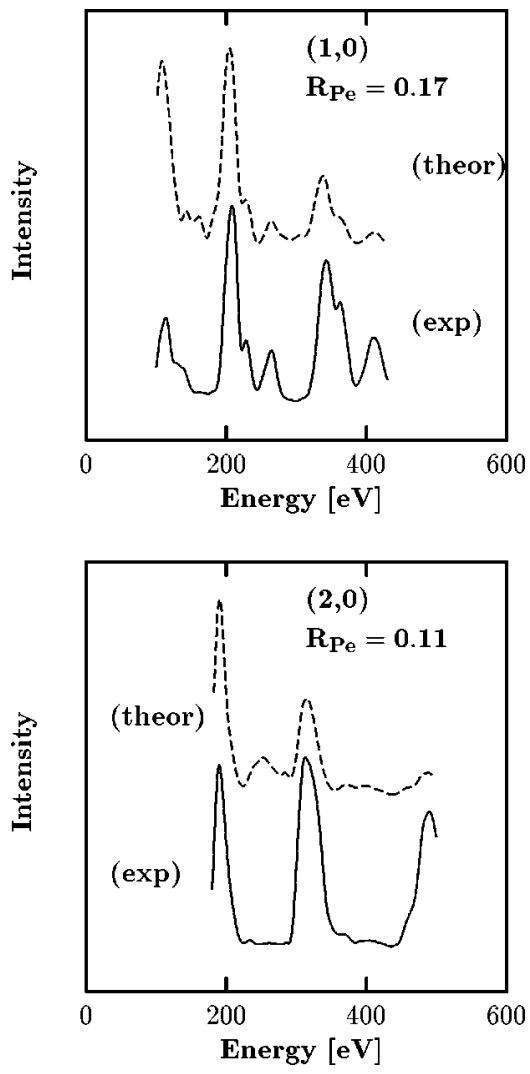

FIG. 6. Best-fit spectra for the $(1,0)$ and $(2,0)$ beam of the thick film compared to the experiment.

sisted of six symmetrically inequivalent beams between 100 $\mathrm{eV}$ and $500 \mathrm{eV}$ yielding $\Delta E=1460 \mathrm{eV}$. During the deposition process, a slight carbon contamination was observed by AES.

As in the case of the thin film, we first tested only models consisting of a single ordered domain. Again, the composition of the top four layers was allowed to be either $\mathrm{Fe}$ or $\mathrm{Au}$. They were assumed to reside on a bcc-Fe(100) bulk, i.e., the actual gold substrate was assumed to be hidden due to electron attenuation. In the structural search, the topmost three layer spacings were varied by up to $\pm 0.2 \AA$ around the corresponding "hard-sphere" values. An uncovered $\mathrm{Fe}(100)$ surface yields the best fit to experiment in this step $(R$ $=0.39$ ). Enforcing a single Au layer covering a Fe(100) surface yields only $R=0.50$, in spite of the fact that AES indicates the presence of some gold within the surface or on top of it. So, a single phase with a full layer of gold floated to the surface as reported by Kellar et al. ${ }^{21}$ for a 15 ML film must

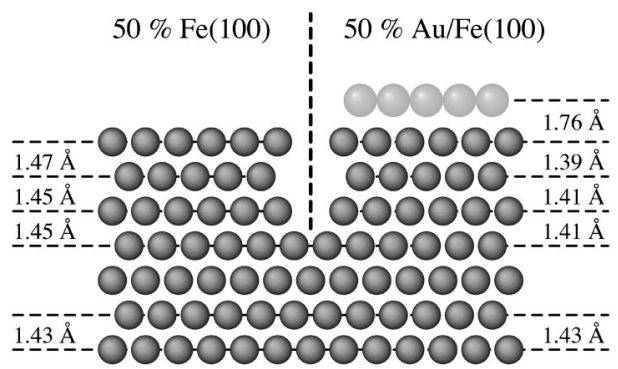

FIG. 7. Visualization of the best-fit structure of a thick Fe film. About half the film is still covered by $\mathrm{Au}(100)$. 
TABLE II. Best-fit parameters of the thick Fe film on $\mathrm{Au}(100)$.

\begin{tabular}{lcc}
\hline \hline & $\mathrm{Fe}(100)$ & $\mathrm{Au} / \mathrm{Fe}(100)$ \\
\hline Surface fraction & $50 \pm 20 \%$ & $50 \mp 20 \%$ \\
$d_{12}$ & $1.47 \pm 0.05 \AA$ & $1.76 \AA \AA_{-0.1}^{+0.2}$ \\
$d_{23}$ & $1.45 \pm 0.03 \AA$ & $1.39 \pm 0.04 \AA$ \\
$d_{34}$ & $1.45 \pm 0.03 \AA$ & $1.41 \pm 0.04 \AA$ \\
$d_{45}$ & $1.43 \pm 0.04 \AA$ & $1.41 \pm 0.04 \AA$ \\
$d_{\text {bulk }} \AA$ & $1.43 \AA$ \\
$a_{v i b}$ (top layer) & $1.43 \AA$ & $0.21 \AA \AA_{-0.06}^{+\infty}$ \\
$a_{v i b}$ (interface Fe) & $0.17 \pm 0.04 \AA$ & $0.15 \AA$ \\
$a_{v i b}$ (bulk Fe) & $0.11 \AA$ & $0.11 \AA$ \\
\hline \hline
\end{tabular}

be ruled out. The observation that the amount of top layer $\mathrm{Au}$ decreases with growing $\mathrm{Fe}$ coverage $\mathrm{e}^{20}$ advises us to consider a model with two domains, one corresponding to a clean and one to a $\mathrm{Au}$ covered $\mathrm{Fe}(100)$ surface. In the corresponding structural search, the top four layer spacings of each domain were varied. Additionally, we allowed for the variation of the spacing in the Fe film below, assuming it to be constant for all layers. Thermal vibrational amplitudes were also adjusted in the course of the fitting procedure. The two-domain model results in a drastic improvement of the fit $(R=0.19)$, the quality of which is demonstrated in Fig. 6 where experimental and best-fit spectra are displayed for two selected beams.

In the best-fit structure, a Au layer still covers about 50\% of the bcc-Fe film as illustrated in Fig. 7. The corresponding best-fit parameters are given in Table II. Interestingly, with $d_{12}=1.76 \AA$ the $\mathrm{Au}-\mathrm{Fe}$ interlayer distance in the Au-covered domain does not show a strong expansion with respect to the hard-sphere value as in the thin-film case. Unfortunately, with values between $1.66 \AA$ and $1.96 \AA$ inside the limits of error, this result must be regarded with caution. The high uncertainty of $d_{12}$ is accompanied by a rather high vibrational amplitude of the top layer Au atoms, $a_{v i b}=0.21 \AA$ probably due to static displacive disorder in the floating $\mathrm{Au}$ layer rather than to simple thermal vibrations. As to the $\mathrm{Fe}$ film underneath that layer, though its top few interlayer distances appear systematically contracted, the bulk value of $d$ $=1.43 \AA$ is always within the limits of error. Any possible film distortion, if present, is smaller than the accuracy of our fit.

The structure of the Au-free part of the film clearly differs from that of a clean $\mathrm{Fe}(100)$ surface. Instead of a contraction (expansion) of the first (second) layer spacings, ${ }^{52,53}$ we find an expanded topmost interlayer distance of $1.47 \AA$ (though the bulk value is within the error limits), deeper spacings are close to the bulk value. Due to the $\mathrm{C}$ contamination mentioned above, this finding does not come as a surprise. Electronegative adsorbates are known to cause a derelaxation of the surface region and expansions of the topmost interlayer distance of bcc (100) surfaces. ${ }^{53-56}$ Thermal vibrational amplitudes, also listed in Table II, all exhibit a trend to relatively high values compared to the value of $0.07 \AA$ expected from the bulk Debye temperature $\left[\Theta_{D}(\mathrm{Fe})=465 \mathrm{~K}\right]$. Again, this indicates the possibility of some positional disorder in the film not present in a well-prepared $\mathrm{Fe}(100)$ single crystal.

Comparing our results to the literature, we note the close agreement between the geometry of the Au-covered domain and the results of Kellar et $a .^{21}$ for a similar film using x-ray photoelectron diffraction. Their model structure to be fitted consisted of a single layer of $\mathrm{Au}$ on top of an unrelaxed bcc-Fe(100) film with an Au-Fe interlayer distance of $d_{12}$ $=1.67 \AA$, which is within the limits of our fit. Yet, this study is based only on Au $4 f$ photoelectron diffraction, "blind" to possible uncovered film areas, unlike the LEED study of Begley et al. ${ }^{17}$ where, however, a domain mixture was not considered and where consequently the simple model of Kellar et al., as in our case, failed to provide a complete description of the film surface.

For the model presented above, we also investigated features such as substitutional disorder, the influence of the $\mathrm{Au}$ bulk below the $\mathrm{Fe}$ film, and the lateral lattice parameter of the Fe film. However, neither attempt yielded any significant improvement over the results already presented. The intermixing expected to occur in the film ${ }^{20}$ is too small to produce a significant effect on the spectra. The Au bulk apparently lies beyond the "information depth" of elastically scattered electrons. The variation of the lateral lattice parameter reproduces that of $\mathrm{Fe}(100)(a=2.866 \AA)$, but the limits of error are too large to exclude the value of $\mathrm{Au}(100)$.

Summarizing, we have shown that a large part of the film surface is covered by a single layer of Au even at this relatively high $\mathrm{Fe}$ coverage. With growing $\mathrm{Fe}$ thickness, Au stays behind in the film ${ }^{20}$ and the "missing" Au atoms are not replaced by Fe atoms incorporated in the Au layer. Instead, the Au layer shrinks into domains opening areas of uncovered bcc-Fe(100). It is this mixture of ordered domains that explains the discrepancies apparent in the literature to date. ${ }^{17,21}$

\section{DISCUSSION AND CONCLUSION}

A surface-active agent, or "surfactant," influences the growth mode of an epitaxial film to improve its structural or morphological properties. In most cases the surfactant consists of a third chemical species. In the present case, film growth is influenced by substrate gold atoms floating to the surface, i.e., atoms from the epitaxial system itself. This allows us to speak of a "self-surfactant" effect ${ }^{16,17,20}$ of Au in the growth of $\mathrm{Fe}$ films on $\mathrm{Au}(100)$. In the following, we will summarize our evidence for its existence, explore its limitations, and give a tentative microscopic model of the growth process.

In the coverage regime up to $1 \mathrm{ML}$, film growth is practically two dimensional rather than three dimensional as expected from the surface free energies of $\mathrm{Au}$ and $\mathrm{Fe}$. Obviously, a segregated layer of $\mathrm{Au}$ is energetically more favorable than exposing $\mathrm{Fe}$ to the vacuum. Yet, the influence of $\mathrm{Au}$ reaches beyond a mere segregation, shaping the $\mathrm{Fe}$ film to the morphology observed in the present paper and rendering Au a true "self-surfactant" in this first growth stage. For the very early stages of Fe film growth, this process has been illuminated in detail in a recent publication, ${ }^{42}$ establishing place exchanges between incoming Fe and toplayer $\mathrm{Au}$ atoms and lateral $\mathrm{Au}$ self-diffusion as the dominant diffusion mechanisms involved. In particular, no lateral diffusion of Fe was required to explain the observed morphology in the nucleation stage. In the present higher coverage case (about $1 \mathrm{ML}$ ), there is a clear tendency of Fe to form subsurface islands with only small amounts of Au admixed, 
rather than a fully homogeneous, disordered distribution of $\mathrm{Fe}$ in the second layer expected for a complete absence of lateral $\mathrm{Fe}$ diffusion. Thus, at some stage, lateral transport of Fe must play a role in the shaping of the films observed in the present paper. Possibly, the higher growth temperature ( $400 \mathrm{~K}$ instead of RT) in our case is responsible for this difference. Alternatively, it is conceivable that the lateral diffusion of $\mathrm{Fe}$ is a comparatively small effect playing no significant role in the initial stages of growth when diffusion lengths required for the formation of Fe "islands" are large.

In contrast, at a coverage of several ML the STM images show a morphology similar to the homoepitaxial growth of $\mathrm{Fe} / \mathrm{Fe}(100),{ }^{29,30}$ i.e., at this stage there is no noticeable "selfsurfactant" effect of Au despite considerable amounts of $\mathrm{Au}$ still floating on the film surface. It seems that the Fe film rather imposes constraints on the floating Au layer, inducing some static displacive disorder and tearing it into small patches. Obviously, the "self-surfactant" effect ends before "bulklike," thick Fe films develop. However, we do not observe its sharp breakdown. STM images of the "wormlike" structures around 2 ML (Sec. III B) still show large, though no longer atomically flat terraces. Thus, the "selfsurfactant" effect of Au on the overall film morphology prevails also in this stage and probably decays gradually until the "thick-film" limit is reached. However, the effect is considerably reduced at 2 ML since it no longer enforces the growth of epitaxial, long-range-ordered Fe films.

In the "wormlike" growth stage, the films apparently consist of localized, elongated defects. From the present data, it is impossible to make quantitative statements on their structure except that the film should be covered by a layer of $\mathrm{Au}$ since this layer is detected by ion scattering spectroscopy. ${ }^{20}$ The apparent height of the "worms" in STM, $\sim 0.6 \AA$, proves that they cannot consist of simple atomic chains positioned atop the outermost $\mathrm{Fe}$ or even $\mathrm{Au}$ layer. Their height corresponds rather to atoms contained in one of the layers, but laterally displaced from the expected hollow position to a bridge or top site. Based on this observation, the following model may serve to explain the origin of the "worms."

Certainly, a bcc(100)-Fe layer of atoms corresponds to a stable arrangement of atoms when part of an iron bulk. However, it is important to notice that in other environments this need not necessarily be true. The most stable free-standing two-dimensional bcc-like arrangement is the quasihexagonal (110) layer with four nearest neighbors per atom, while in a bcc(100) plane each atom has only next-nearest and no nearest neighbors at all-the latter are all found in the adjacent layers. Thus, the initially observed $\mathrm{Fe}(100)$ monolayer is mainly stabilized by the two surrounding Au layers. Somewhere beyond monolayer coverage, this stabilization becomes insufficient. Assuming the nearest-neighbor bonding between two $\mathrm{Fe}$ atoms to be strongest in this system, the formation of in-plane nearest-neighbor Fe bonds through lo- cal displacements could be energetically favorable at the expense of Au-Fe interlayer bonding. Defect structures, such as those observed in the present paper, are a consequence of this mechanism. For example, one might imagine the "worms" to consist of short chains of Fe atoms pushed into the bcc(100) layer in a local, bcc(110)-like arrangement. These atoms would reside on bridge sites of the layer below, consistent with the height of the "worms."

The proposed mechanism also explains two other observations. First, the $\operatorname{bcc}(100)$ arrangement would face strong lateral distortions around (110)-like defect chains, extending into the adjacent layers as well. This explains the observed rapid vanishing of the LEED pattern as the lateral periodicity is broken in several layers. Second, from the LEED pattern we find that thick films keep the (100) orientation of the $\mathrm{Au}$ bulk. This is only possible if the interface between $\mathrm{Fe}$ and $\mathrm{Au}$ retains some of the periodicity of the $\mathrm{Au}(100)$ bulk, so at this stage a nearly amorphous interface is out of the question. Our model implies that the "wormlike" defect structure is energetically favorable when not enough $\mathrm{Fe}$ is available to create a bulklike Fe environment. With the deposition of more $\mathrm{Fe}$, these defects are no longer advantageous, and it is well possible that a partial reincorporation of the "worm" atoms into the bcc lattice takes place, largely restoring the periodicity at the interface.

In summary, we have shown that the growth of Fe films on $\mathrm{Au}(100)$ begins with the formation of large and flat terraces of Fe covered by a floating layer of $\mathrm{Au}$. A "selfsurfactant" effect of Au induces the flat growth of the Fe layer, though some deviations from pure monolayer growth occur. Above a certain coverage ( $\geqslant 1 \mathrm{ML}$ ), the film structure is dominated by large amounts of short, linear defects, possibly due to the formation of $\mathrm{Fe}-\mathrm{Fe}$ bonds at the expense of the Au-Fe interlayer bonding. Yet, on a large scale the film still consists of flat terraces, indicating that the $\mathrm{Au}$ "selfsurfactant" effect on the film morphology decays only gradually in this stage. In much thicker films, the long-range order of the Fe lattice is re-established, and a bcc(100)-Fe film characterized by small terraces forms. Our results imply that the growth of a flat and defect-free bcc-Fe film on $\mathrm{Au}(100)$ remains a major challenge to current experimental techniques. The assumption of perfect film growth implicit in many studies of nonstructural (magnetic, optical) properties of Fe/Au film and multilayer systems may often not be justified.

\section{ACKNOWLEDGMENTS}

This work was supported by the Acciones Integradas Program of the DAAD and the Ministerio of Educación y Cultura. The research in Spain has also been financed by the CICyT through Project No. PB-95-454324 as well as partly through Project No. PB97-0031. Support by the Deutsche Forschungsgemeinschaft (DFG) is also gratefully acknowledged. 
${ }^{1}$ K. Baberschke, Appl. Phys. A: Mater. Sci. Process. 62, 417 (1996).

${ }^{2}$ J.J. de Miguel, A. Cebollada, J.M. Gallego, R. Miranda, C.M Schneider, P. Schuster, and J. Kirschner, J. Magn. Magn. Mater. 93, 1 (1991).

${ }^{3}$ S.S.P. Parkin, Z.G. Li, and D.J. Smith, Appl. Phys. Lett. 58, 2710 (1991).

${ }^{4}$ H.A. van der Vegt, H.M. van Pinxteren, M. Lohmeier, E. Vlieg, and J.M.C. Thornton, Phys. Rev. Lett. 68, 3335 (1992).

${ }^{5}$ P. Schieffer, C. Krembel, M.C. Hanf, and G. Gewinner, Phys. Rev. B 57, 1141 (1998).

${ }^{6}$ R. Opitz, S. Löbus, A. Thissen, and R. Courths, Surf. Sci. 370, 293 (1997).

${ }^{7}$ K. Shintaku, Y. Daitoh, and T. Shinjo, Phys. Rev. B 47, 14584 (1993).

${ }^{8}$ S.N. Okuno and K. Inomata, Phys. Rev. B 51, 6139 (1995).

${ }^{9}$ K. Inomata, S.N. Okuno, Y. Saito, and K. Yusu, J. Magn. Magn. Mater. 156, 219 (1996).

${ }^{10}$ T. Katayama, Y. Suzuki, and W. Geerts, J. Magn. Magn. Mater. 156, 158 (1996).

${ }^{11}$ K. Sato, J. Abe, H. Ikekame, K. Takanashi, S. Mitani, and H. Fujimori, J. Magn. Soc. Jpn. 20, 35 (1996).

${ }^{12}$ K. Takanashi, S. Mitani, M. Sano, H. Fujimori, H. Nakajima, and A. Osawa, Appl. Phys. Lett. 67, 1016 (1995).

${ }^{13}$ S. Mitani, K. Takanashi, H. Nakajima, K. Sato, R. Schreiber, P. Grünberg, and H. Fujimori, J. Magn. Magn. Mater. 156, 7 (1996).

${ }^{14}$ B. Predel, in Zahlenwerte und Funktionen aus Naturwissenschaft und Technik, edited by O. Madelung, Landolt-Börnstein, New Series, Group IV, Vol. 5, Pt. a (Springer-Verlag, Berlin, 1991).

${ }^{15}$ S. Bader and E.R. Moog, J. Appl. Phys. 61, 3729 (1987), and references therein.

${ }^{16}$ F.J. Himpsel, Phys. Rev. B 44, 5966 (1991).

${ }^{17}$ A.M. Begley, S. K. Kim, J. Quinn, F. Jona, H. Over, and P.M. Marcus, Phys. Rev. B 48, 1779 (1993).

${ }^{18}$ Y.-L. He and G.-C. Wang, Phys. Rev. Lett. 71, 3834 (1993).

${ }^{19}$ S. De Rossi, F. Ciccacci, and S. Crampin, Phys. Rev. B 52, 3063 (1995).

${ }^{20}$ C.J. Pastor, C. Limones, J.J. Hinarejos, J.M. García, R. Miranda, J. Gómez-Goñi, J.E. Ortega, and H.D. Abruña, Surf. Sci. 364, L505 (1996).

${ }^{21}$ S.A. Kellar, Y. Chen, W.R.A. Huff, E.J. Moler, Z. Hussain, and D.A. Shirley, Phys. Rev. B 57, 1890 (1998).

${ }^{22}$ L.Z. Mezey and J. Giber, Jpn. J. Appl. Phys., Part 1 21, 1569 (1982).

${ }^{23}$ R. Germar, W. Dürr, J.W. Krewer, D. Pescia, and W. Gudat, Appl. Phys. A: Solids Surf. 47, 393 (1988).

${ }^{24}$ Y. Suzuki, T. Katayama, S. Yoshida, K. Tanaka, and K. Sato, Phys. Rev. Lett. 68, 3355 (1992).

${ }^{25}$ Q. Jiang, A. Chan, and G.-C. Wang, Phys. Rev. B 50, 11116 (1994).

${ }^{26}$ T. Kawagoe, K. Nakamura, T. Terashima, H. Kaihoko, and T. Mizoguchi, J. Magn. Magn. Mater. 148, 185 (1995).
${ }^{27}$ Q. Jiang, Y.-L. He, and G.-C. Wang, Surf. Sci. 295, 197 (1993).

${ }^{28}$ J. de la Figuera, J.E. Prieto, G. Kostka, S. Müller, C. Ocal, R. Miranda, and K. Heinz, Surf. Sci. 349, L139 (1996).

${ }^{29}$ J.A. Stroscio, D.T. Pierce, and R.A. Dragoset, Phys. Rev. Lett. 70, 3615 (1993).

${ }^{30}$ J.A. Stroscio, D.T. Pierce, M.D. Stiles, A. Zangwill, and L.M. Sander, Phys. Rev. Lett. 75, 4246 (1995).

${ }^{31}$ J.M. García, O. Sanchez, P. Segovia, J.E. Ortega, J. Alvarez, A.L. Vázquez de Parga, and R. Miranda, Appl. Phys. A: Mater. Sci. Process. 61, 609 (1995).

${ }^{32}$ K. Heinz, Rep. Prog. Phys. 58, 637 (1995).

${ }^{33}$ H. Wedler and K. Heinz, Vak. Forsch. Prax. 7, 107 (1995).

${ }^{34}$ J.B. Pendry, J. Phys. C 13, 937 (1980).

35 M.A. van Hove and S.Y. Tong, Surface Crystallography by LEED (Springer, Berlin, 1979).

${ }^{36}$ Y. Gauthier, Y. Joly, R. Baudoing, and J. Rundgren, Phys. Rev. B 31, 6216 (1985).

${ }^{37}$ M. Kottcke and K. Heinz, Surf. Sci. 376, 352 (1997).

${ }^{38}$ D.G. Fedak and N.A. Gjostein, Surf. Sci. 8, 77 (1967).

${ }^{39}$ G. Binnig, H. Rohrer, C. Gerber, and E. Stoll, Surf. Sci. 144, 321 (1984).

${ }^{40}$ S.G.J. Mochrie, D.M. Zehner, B.M. Ocko, and D. Gibbs, Phys. Rev. Lett. 64, 2925 (1990).

${ }^{41}$ O.S. Hernán, J.M. Gallego, A.L. Vázquez de Parga, and R. Miranda, Appl. Phys. A: Mater. Sci. Process. 66, S1117 (1998).

42 O.S. Hernán, A.L. Vázquez de Parga, J. M. Gallego, and R. Miranda, Surf. Sci. 415, 106 (1998).

${ }^{43}$ K. Heinz, P. Bayer, and S. Müller, Surf. Rev. Lett. 2, 89 (1995).

${ }^{44}$ C. Rath, J.E. Prieto, S. Müller, R. Miranda, and K. Heinz, Phys. Rev. B 55, 10791 (1997).

${ }^{45}$ J.E. Prieto, C. Rath, S. Müller, R. Miranda, and K. Heinz, Surf. Sci. 401, 248 (1998).

${ }^{46}$ S.C. Wu, H. Li, J. Quinn, D. Tian, Y.S. Li, A.M. Begley, S.K. Kim, F. Jona, and P.M. Marcus, Phys. Rev. B 49, 8353 (1994).

${ }^{47}$ V. Fiorentini, M. Methfessel, and M. Scheffler, Phys. Rev. Lett. 71, 1051 (1993).

${ }^{48}$ K. Heinz and G. Besold, Surf. Sci. 125, 515 (1983).

${ }^{49}$ N. Nakayama, T. Okuyama, and T. Shinjo, J. Phys.: Condens. Matter 5, 1173 (1993).

${ }^{50}$ Z.P. Shi, J.F. Cooke, Z. Zhang, and B.M. Klein, Phys. Rev. B 54, 3030 (1996)

${ }^{51}$ J.-T. Wang, Z.-Q. Li, and Y. Kawazoe, J. Phys.: Condens. Matter 9, 4549 (1997).

${ }^{52}$ Z.Q. Wang, Y.S. Li, F. Jona, and P.M. Marcus, Solid State Commun. 61, 623 (1987).

${ }^{53}$ R.L. Headrick, P. Konarski, S.M. Yalisove, and W.R. Graham, Phys. Rev. B 39, 5713 (1989).

${ }^{54}$ F. Jona and P.M. Marcus, Solid State Commun. 64, 667 (1987).

${ }^{55}$ D. Jentz, S. Rizzi, A. Barbieri, D. Kelly, M.A. van Hove, and G.A. Somorjai, Surf. Sci. 329, 14 (1995).

${ }^{56}$ M. Sporn, E. Platzgummer, M. Pinczolits, W. Hebenstreit, M. Schmid, W. Hofer, and P. Varga, Surf. Sci. 396, 78 (1998). 